\title{
Predictive pattern classification can distinguish gender identity subtypes from behavior and brain imaging
}

Benjamin Clemens ${ }^{1,2}$, Birgit Derntl ${ }^{3,4,5}$, Elke Smith ${ }^{1}$, Jessica Junger ${ }^{1,2}$, Josef Neulen ${ }^{6}$, Gianluca Mingoia $^{7}$, Frank Schneider ${ }^{1,2}$, Ted Abel ${ }^{8,9}$, Danilo Bzdok ${ }^{1,2,10,11,12^{*}}$, Ute Habel ${ }^{1,2 *}$

\footnotetext{
${ }^{1}$ Department of Psychiatry, Psychotherapy and Psychosomatics, Faculty of Medicine, RWTH Aachen, Pauwelsstr. 30, 52074 Aachen, Germany.

${ }^{2}$ Institute of Neuroscience and Medicine: JARA-Institute Brain Structure Function Relationship (INM 10), Research Center Jülich, Jülich, Germany.

${ }^{3}$ Department of Psychiatry and Psychotherapy, University of Tübingen, Calwerst. 14, 72076 Tübingen, Germany.

${ }^{4}$ Werner Reichardt Center for Integrative Neuroscience (CIN), University of Tübingen, Otfried-Müller-Str. 25, 72076 Tübingen, Germany.

${ }^{5}$ LEAD Graduate School and Research Network, Gartenstr. 29a, 72074 Tübingen, Germany.

${ }^{6}$ Department of Gynecological Endocrinology and Reproductive Medicine, Faculty of Medicine, RWTH Aachen University, Pauwelsstr. 30, 52074 Aachen, Germany.

${ }^{7}$ Interdisciplinary Center for Clinical Research (IZKF), RWTH Aachen University, Pauwelsstr. 30, 52074 Aachen, Germany.

${ }^{8}$ Department of Biology, University of Pennsylvania, Philadelphia, United States.

${ }^{9}$ Department of Molecular Physiology and Biophysics, Iowa Neuroscience Institute, Carver College of Medicine, University of Iowa, Iowa City, Iowa, United States.

${ }^{10}$ Parietal Team, INRIA/Neurospin Saclay, France.

${ }^{11}$ Department of Biomedical Engineering, Faculty of Medicine, McGill University, Montreal, Canada.

${ }^{12}$ Montreal Institute for Learning Algorithms (MILA), Montreal, Canada.
}

* Those authors contributed equally to the present study.

Corresponding author:

Dr. Benjamin Clemens

Pauwelsstrasse 30, 52074 Aachen, Germany

0049-2418089407; bclemens@ukaachen.de

Short title: Defining gender in the brain with machine learning

Keywords: fMRI; resting-state functional connectivity; transgender; transsexualism; machine learning 


\section{Abstract}

Mis en forme : Gauche

The exact neurobiological underpinnings of gender identity (i.e. the subjective perception of oneself belonging to a certain gender), still remain unknown. Combining both resting-state functional connectivity and behavioral data, we examined gender identity in cis- and transgender persons using a data-driven machine-learning strategy. Intrinsic functional connectivity and questionnaire data were obtained from cisgender (men/women) and transgender (trans men/trans women) individuals. Machine-learning algorithms reliably detected gender identity with high prediction accuracy in each of the four groups based on connectivity signatures alone. The four normative gender groups were classified with accuracies ranging from $48 \%$ to $62 \%$ (exceeding chance level at $25 \%$ ). These connectivity based classification accuracies exceeded those obtained from a widely established behavioral instrument for gender identity. Using canonical correlation analyses, functional brain measurements and questionnaire data were then integrated to delineate nine canonical vectors (i.e., brain-gender axes), providing a multi-level window into the conventional sex dichotomy. Our dimensional gender perspective captures four distinguishable brain phenotypes for gender identity, advocating a biologically grounded re-conceptualization of gender dimorphism. We hope to pave the way towards objective, data-driven diagnostic markers for gender identity and transgender, taking into account neurobiological and behavioral differences in an integrative modeling approach. 


\section{Introduction}

Although visible anatomy and gender identity are identical in most individuals, there are exceptions. Throughout the manuscript, we will use the term "biological sex" to describe the sex assigned to each human being at birth, based on the anatomy of the reproductive system. The term "gender identity" will be used to describe the subjective perception of oneself belonging to a certain gender, which can be male, female, neither or a combination of both. Different terms have been used to describe persons whose gender identity does not align with their biological sex. Of all these terms, "transgender" has become the preferred term, whereas "cisgender" refers to persons whose biological sex aligns with their gender identity. Transgender individuals can be either trans men (Tm), describing individuals born with the biological sex of a woman but identify as men, or trans women (Tw), describing individuals with female gender identity but male biological sex. An additional term, which has been used in several classification systems but is now being discarded, is "gender dysphoria". This mental health diagnosis entails severe discomfort arising from the discrepancy between gender identity and biological sex (American Psychiatric Association 2013). Importantly, being transgender does not equate with a mental health condition and not all transgender persons suffer from gender dysphoria (Safer and Tangpricha 2019). To reduce stigma and facilitate access to relevant health care needs for transgender persons, the plan for the ICD-11 is to add gender incongruence to the sexual health section and remove gender dysphoria entirely (Reed et al. 2016). In accordance with this fundamental change in terminology, we will also avoid the term gender dysphoria and use the term gender incongruence throughout the manuscript.

According to a recent estimate, more than 1.45 million transgender individuals, representing at least $0.6 \%$ of the overall population, live in the U.S.A. alone (Flores et al. 2016). Prevalence rates, however, might have been severely underestimated (Mędraś and Jóźków 2010). Most likely, these numbers will continue to increase in the near future due to changes in 
legislation, increased visibility of transgender celebrities in the media, increased availability of medical procedures and decreased stigmatization. One of the most severe problems for transgender persons are the numerous barriers to accessing appropriate and competent medical care (Safer and Tangpricha 2019). These challenges in accessing health care services, a lack of knowledgeable primary care providers and societal stigmatization have been shown to contribute to multiple health issues in transgender persons: substance abuse, mental health conditions, increased rates of certain types of cancer, infections, and chronic diseases (Jaffee et al. 2016; Reisner et al. 2016). This exemplifies the need for clinicians and scientists to further increase our understanding of the specific medical issues and underlying neurobiological mechanisms that are relevant to this population.

Recent works also suggests the existence of a biological underpinning to gender identity that is present already at birth (Safer et al. 2008; Saraswat et al. 2015), with twin studies demonstrating greater concordance with regard to transgender identity for identical twins as compared to fraternal twins (Heylens et al. 2012). Furthermore, the sex hormone androgen seems to play an important role. People who were exposed to excess androgen in utero show increased rates of male gender identity (Dessens et al. 2005), whereas individuals with androgen insensitivity syndrome exhibit increased rates of female gender identity (Mazur 2005). So how exactly do transgender individuals differ from cisgender individuals with respect to brain morphology, connectivity and functioning? This question seems relevant from a scientific, societal and clinical standpoint. The scarce neuroscience findings have pulled the discussion in different directions: data from functional magnetic resonance imaging (fMRI) studies provide a complex pattern of results and are often based on small samples, which have mostly not been replicated and in many cases involve studies with trans women only (Smith et al. 2015; Mueller et al. 2017). While earlier post mortem studies indicated a feminization of hypothalamic nuclei and the bed nucleus of the stria terminalis in Tw (Zhou et al. 1995), a more complex pattern 
emerges when also incorporating results from later post mortem studies investigating transgender subjects (Garcia-Falgueras and Swaab 2008; Garcia-Falgueras and Swaab 2009). Overall, there is a common thought pattern that hinders insights from psychological and neuroscientific studies of transgender: Traditional approaches have routinely assumed two strict gender categories, thus reinforcing a sexually dimorphic view of human behavior and cognition.

In contrast, our study strives for a dimensional across-group perspective on the neural basis of transgender. Using a comprehensive sample of men, women, trans men and trans women, we leveraged machine-learning algorithms to elucidate the complex interactions between gender identity and biological sex, thereby defining multidimensional markers that delineate gender phenotypes across cisgender and transgender. The overarching goal of our approach is to better understand the neurobiological basis of transgender by searching for biobehavioral markers instead of relying simply on primary sexual characteristics. Considering accumulating evidence for more nuanced views on gender and its manifestation in the human brain (Joel et al. 2015; Manzouri et al. 2017; Manzouri and Savic 2018), one might argue that there may be no such thing as a categorically male or a female brain. Using this strictly categorical approach, there has been little progress towards answering an important question, which resides at the core of all neurobiologically oriented transgender research: do trans men and trans women represent separate and dissociable subtypes of gender, or can we classify all people as either male or female? For the present study, we employ a data-driven, machine learning approach, hoping to answer the aforementioned question and shed light on the neurobiological underpinnings of transgender and gender incongruence. 


\section{Methods}

Participants

92 participants were recruited in the present study, including 23 cisgender males, 23 cisgender females, 23 trans men and 23 trans women. All cisgender participants were recruited via public announcement around Aachen (Germany). Tm and Tw were recruited in self-help groups and at the Department of Gynaecological Endocrinology and Reproductive Medicine of the RWTH Aachen University Hospital, Germany. All transgender participants declared their intention of undergoing cross-sex hormone therapy in the future, expressed a strong sense of belonging to the opposite sex and lived the desired role in everyday life. Furthermore, all transgender participants fulfilled diagnostic criteria for gender dysphoria, as diagnosed by a board-certified mental-health professional. The German version of the Structured Clinical Interview of the fourth edition of the Diagnostic and Statistical Manual of Mental Disorders (DSM-IV) (Wittchen et al. 1997) was used to ensure the exclusion of participants with mental disorders unrelated to gender incongruence. For all cisgender and transgender participants, further exclusion criteria were neurological disorders, other medical conditions affecting the brain metabolism, and first degree relatives with a history of mental disorders. The local Ethics Committee of the Medical Faculty of RWTH Aachen University approved the study (EK 088/09). Participants were financially reimbursed and gave their written informed consent for participation in the current study.

Table 1 - Demographic information in the participant sample

\begin{tabular}{|c|c|c|c|c|}
\hline & Female (F) & Male (M) & Trans men (Tm) & $\begin{array}{c}\text { Trans women } \\
\text { (Tw) }\end{array}$ \\
\hline Age & $32(11)$ & $32(9)$ & $25(7)$ & $31(10)$ \\
\hline Years of education & $15(3)$ & $15(3)$ & $14(2)$ & $14(3)$ \\
\hline Biological Sex & 46 & 46 & n.a. & n.a. \\
\hline Gender Identity & 23 & 23 & 23 & 23 \\
\hline
\end{tabular}


The table gives relevant demographic information (means, with standard deviations in brackets) about participants, divided by groups. Since the term biological sex refers to the anatomically determined sex, which does not include transgender sub-groups, the respective cells in the table remain empty.

\section{Procedures}

Our dataset consisted of two parts: questionnaire data assessing self-perceived gender identity and resting-state fMRI scans. In recent years, functional connectivity fMRI approaches found several brain regions whose spontaneous low-frequency fluctuations $(<0.1 \mathrm{~Hz})$ of the blood oxygen level-dependent (BOLD) signal registered during resting-state correlate with each other. Those regions are believed to be functionally connected to each other in the absence of any particular task constraints, reflecting the brain's intrinsic functional architecture (Biswal et al. 2010; Smith et al. 2009). Furthermore, our data included the detailed, quantified information regarding gender identity, which is offered by the Bem Sex-Role Inventory (BSRI; (Bem 1974)) (cf. methods). This questionnaire asks participants to rate themselves on culturally desirable male and female traits. The BSRI is one of the very few empirically based questionnaires to examine gender identity, with the resulting gender categorizations correlating with various stereotypical gendered behaviors (Bem 1977; Lee 1982; Hoffman and Borders 2001; Savic and Arver 2011). While several psychometric instruments have been specifically designed for the assessment of gender identity in transgender individuals, we did not choose one of these specialized questionnaires here because this would have hindered directly comparing and jointly analyzing cis- and transgender individuals. Studies reporting results about the application of the BSRI to transgender populations remain rare. However, several previous studies, conducted in Spain (Gòmez-Gill et al. 2012) and Poland (Herman-Jeglinska et al. 2002), have employed the BSRI to compare cisgender controls and transgender individuals. Both reports revealed that transgender individuals scored on the BSRI as a function 
of their gender identity instead of their biological sex. These results substantiate the BSRI as a useful measure of gender identity, also in transgender individuals.

\section{Brain imaging data acquisition}

Using a 3 Tesla Siemens Trio MR Scanner (Siemens Medical Systems, Erlangen, Germany) at the Department of Psychiatry, Psychotherapy and Psychosomatics of the RWTH Aachen University Hospital the following sequences covering the entire brain were obtained for each participant: a) 4 min T1-weighted MP-RAGE 3D measurement (TR=1900, TE=2.52, $\mathrm{TI}=900 ; \alpha=9^{\circ}, \mathrm{FoV}=250 \mathrm{~mm}^{2}$, voxel size: $1 \times 1 \times 1 \mathrm{~mm}^{3}$, slices=176) and b) a $6.2 \mathrm{~min} \mathrm{~T} 2 *_{-}$ weighted echo-planar imaging (EPI) resting state condition $\left(\mathrm{TR}=3000, \mathrm{TE}=35, \alpha=84^{\circ}\right.$, FoV=192 mm, voxel size: $3 \times 3 \times 3 \mathrm{~mm}^{3}, 44$ slices, gap 15\%, 64×64 matrix, repetitions=124). For the resting state condition, participants were asked to relax in the scanner, keep their eyes open and avoid falling asleep, which we confirmed in post-scan interviews.

\section{Image processing}

Pre-processing of the fMRI data was carried out using a standard pipeline, including spatial and temporal data normalization and accounting for in-scanner head motion. All preprocessing was done with FEAT (FMRI Expert Analysis Tool) Version 6.00, part of FSL (FMRIB's Software Library). We discarded the first three images of each functional series to avoid T1 saturation effects; the remaining 121 volumes were utilized. The following signal processing was applied; motion correction using MCFLIRT (Jenkinson et al. 2002), non-brain removal using BET (Smith 2002), spatial smoothing using a Gaussian kernel of FWHM 6.0mm, grand-mean intensity normalization of the entire 4D dataset by a single multiplicative factor, high-pass temporal filtering (Gaussian-weighted least-squares straight line fitting, with 
sigma $=360$ s). Low-pass filtering was avoided to preserve high-frequency content and keep the largest frequency range. Registration to high resolution structural and/or standard space images was carried out using FLIRT (Jenkinson and Smith 2001). Registration from high resolution structural to standard space was then refined using FNIRT nonlinear registration.

Additional preprocessing steps included masking of non-brain voxels, voxel-wise demeaning of the data, and normalization of data to MNI space. To further reduce spurious correlations associated with head-motion in all 92 participants, variance that could be explained by head motion was removed from each voxel's time series. Adhering to previously published studies (Chai et al. 2012; Kernbach et al. 2018; Satterthwaite et al. 2013), we helped remove nuisance signals based on 24 regressors of no interest: a) the 6 motion parameters derived from the image realignment, b) their 6 first derivatives, and c) their respective 12 squared terms. This regression approach has been shown to increase specificity and sensitivity of functional connectivity analyses and to detect valid signal correlation at rest (Satterthwaite et al. 2013).

Statistical analysis: pattern classification of four gender groups from brain connectivity

Please also refer to Figure 1 for a schematic overview of all statistical analyses conducted for the present study. First, we aimed to classify correlated patterns of functional connectivity and behavioral data that can simultaneously appreciate our four gender groups men, women, trans men and trans women. To this end, we used maximum-margin linear support vector machines (SVM; C hyper-parameter set to default) to reveal whether a 6-minute restingstate fMRI scan enables grouping of participants reflecting gender identity. SVMs were chosen because they are probably the most pervasively used pattern classification algorithm in bioinformatics in general (Hastie et al., 2001), with many previous applications in the neuroimaging domain (e.g. Hanson et al., 2008). To avoid overfitting, we re-expressed the 
whole-brain time-series maps in a quintessential network representation as effective summary of distributed data variation. First, independent component analysis (ICA) was conducted at the group level based on a concatenated subsample of functional maps (25 fMRI scans randomly drawn for each of the participants) and then, 50 spatiotemporal network units (Bzdok et al. 2016) were extracted for further analysis (preserving blindness to class labels, cf. Hastie et al., 2001). Second, functional connectivity was computed as linear correlation between each pair of the extracted ensemble of 50 distributed macroscopic networks. This approach yielded 1,225 unique network-network coupling relations in each participant. Third, these 1,225 subjectspecific measures of between-network communication were further reduced using principal component analysis (PCA) to obtain 50 expressions of the main underlying directions of functional network variation idiosyncratic to each participant.

To obtain an unbiased estimate of the expected performance in future brain-imaging data, cross-validation was performed in 100 random, but group-balanced data splits (Witten et al. 2009). $95 \%$ of the data were used for model training and $5 \%$ of the data for model evaluation of prediction performance. The analysis strategy provides insight into how the neural signature underlying male vs. trans men vs. female vs. trans women trade off against each for prediction at the single-subject level. Specifically, predictive models were derived in a one-versus-rest scheme were each sub-type was classified against the three remaining gender sub-types (Hastie et al., 2001). Thus, we were able to quantify, for instance, how many Tm were falsely recognized to belong to the male, female or Tw groups. 

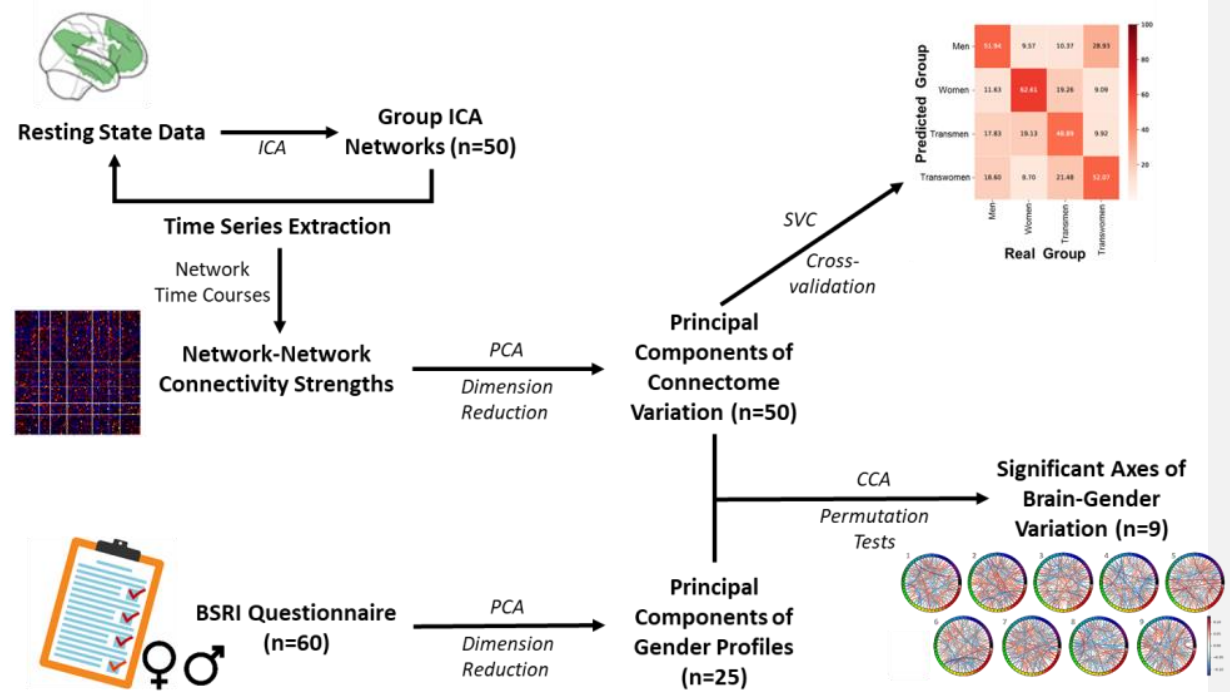

\section{Figure 1 - Overview of the analysis workflow}

Depicts the flow of analyses for both functional imaging and behavioral data. After standard preprocessing, resting-state scans of all 92 participants were subjected to independent component analysis (ICA) and time series extraction to obtain spatiotemporally coherent networks. The resulting time courses of each ensuing network were used to compute between-network functional connectivity strengths. We then applied dimensionality reduction via principal component analysis (PCA) to these connectivity indices. The same dimension reduction procedure was separately applied also to the behavioral data, i.e. scores of each participant for the 60 items of the Bem Sex-Role Inventory (BSRI). In this way, we obtained 50 principal components of functional connectivity and 25 principal components of BSRI items. On the one hand, the essential functional coupling components were fed into the maximum-margin linear support vector classifier (SVC), which after cross-validation resulted in the values for prediction accuracies to be expected in individuals assessed in the future, as depicted in Figure 2. In a separate analysis of our study, both BSRI and functional connectivity components were jointly fed into canonical correlation analysis (CCA). By means of 1,000 permutation iterations, allowing for a best-possible significance threshold of 0.001, a null distribution of random association between brain network dynamics and behavioral gender tendencies across individuals was obtained. $P$ - 
values were derived from the number of correlations $r$ that exceeded the null CCA model and this nonparametric hypothesis testing procedure yielded $k=9$ statistically significant CCA modes. These significant variants of brain-gender association are depicted in Figure 3a. Explicit correction for multiple comparisons was carried out searching through all estimated CCA modes (all $P<0.001$, family-wise-error-corrected).

Statistical analysis: extracting continuous brain-gender phenotypes

Subsequently, we sought to assess the feasibility of extracting continuous brainbehavior associations underlying gender variability. We acquired detailed information regarding gender identity and gender role, which is offered by the BSRI (Bem 1974). The BSRI constitutes one of the very few empirically based questionnaires to examine how people identify themselves with respect to gender (Lee 1982; Hoffman and Borders 2001). Designed to facilitate research on the concept of psychological androgyny, the BSRI presents participants with 60 different personality traits which they rate themselves on using a 7-point Likert scale, ranging from 1 (never or almost never true) to 7 (always or almost always true). Among these 60 traits, 20 are stereotypically masculine (e.g., forceful, dominant), 20 are stereotypically feminine (e.g., affectionate, sympathetic), and 20 are described as 'filler traits' thought to be gender neutral. Both the typical masculine and the typical feminine traits in the BSRI represent culturally desirable traits for men and women, respectively, while 10 of the gender-neutral items were conceptualized as desirable for both sexes (e.g., adaptable, sincere) and the other 10 were undesirable for both sexes (e.g., inefficient, jealous) (Bem 1974). Attempting gender assignments based on behavioral traits captured by the BSRI, we used the original median-split scoring procedure (Bem 1977). We used the German version of the BSRI, which has been shown to possess good validity and reliability in a population sample totaling 580 German men and women (Schneider-Düker and Kohler 1988). For the canonical correlation analyses (CCA) 
reported here, we used the raw scores for each single item of the BSRI for each individual participant.

Across all 92 participants, we sought dominant coupling regimes - "modes" of population variation - that provide insight into how functional variation inside functional networks can explain how individuals vary in gender-characteristic behavior. An important advantage of resting-state fMRI data, as compared to task-based fMRI, is that it is not influenced by the constraints of a specific task that might confound results with respect to gender. Combining both variable sets (BSRI \& resting-state fMRI) in the context of the CCA, will give us an innovative original combination of self-assessment questionnaire and neurobiological data on which we will demonstrate the feasibility of a continuous brain-gender axis across the four participant groups. CCA is a natural choice of method to interrogate such a multivariate correspondence between two high-dimensional variable sets. For the CCA, the same connectomic information was used as in the aforementioned SVM analyses. A first variable set $\mathrm{X}$ corresponded to the subject-specific between-network communication measures (92 x 50 matrix) and a second variable set $\mathrm{Y}$ was constructed from the between-network coupling strengths (92 x 25 BSRI items, reduced by PCA to avoid overfitting). CCA involves finding the canonical vectors $\mathrm{u}$ and $\mathrm{v}$ that maximize the symmetric relationship between a linear combination of functional network connectivity $(\mathrm{X})$ and a linear combination of gender facets (Y). The multivariate pattern learning technique thus reveals a series of functional connectivity modes, each of which identifies the two projections $\mathrm{Xu}$ and $\mathrm{Yv}$ that yielded maximal linear cooccurrence between sets of large-scale network couplings and sets of behavioral tendencies across our four gender groups.

Each estimated brain-gender mode was assessed for statistically significant robustness as determined by hypothesis testing in a permutation procedure used in previous research (Miller et al. 2016). Relying on minimal modeling assumptions, a null distribution was derived 
for the achieved correlation between canonical variations resulting from CCA analysis. In 1,000 permutation iterations, corresponding to a best-possible significance threshold of 0.001 , the between-network connectivity matrix was held constant, while the BSRI questionnaire items were subject to participant-wise random shuffling. The constructed surrogate data preserved the statistical structure idiosyncratic to the functional network relationships, yet permitted to selectively destroy the signal property related to the gender behavior. The distribution generated here from reflected the null hypothesis of random association between brain network dynamics and behavioral gender tendencies across individuals. Pearson's correlation coefficient between the perturbed canonical vectors of the network side and the behavior side was recorded in each iteration to obtain a null distribution of the test statistic. P-values were obtained from the number of correlations $r$ that exceeded the null CCA model (Miller et al. 2016). This nonparametric hypothesis testing procedure yielded k=9 highly significant CCA modes, where explicit correction for multiple comparisons was carried out searching through all estimated CCA modes (all $\mathrm{P}<0.001$, family-wise-error-corrected). Our CCA analysis pipeline therefore directly follows several previous studies (Smith et al. 2015; Miller et al. 2016; Kernbach et al. 2018). Analogous to this research, the present validation using null-hypothesis permutation testing built confidence that our modes of brain-behavior variation are statistically significant and unlikely explained by noise. 


\section{Results}

All participants were right-handed native German speakers, except for one left-handed participant in each of the four groups. Handedness was assessed by means of the Edinburgh Handedness Inventory (Oldfield 1971). Participants took part in two functional MR tasks, which were partly reported elsewhere (Junger et al. 2014). The four groups did not differ significantly regarding years of education $(\mathrm{F}=1.158, \mathrm{p}=.331)$, but there was a small group difference with respect to age $(\mathrm{F}=3.151, \mathrm{p}=.029)$ (see Table 1). This was due to the younger age of the trans men group, as compared to all other three groups.

Machine-learning algorithms were able to accurately distinguish men $(\mathrm{n}=23)$, women $(n=23)$, trans men $(n=23)$ and trans women $(n=23)$ (see Table 1 for demographic info) based on brain scans of intrinsic functional connectivity. As a first step, we assessed the usefulness of our brain-based approach for classifying male and female participants, comparing our pattern classification results with those from the BSRI. Using BSRI data alone enabled us to classify male and female participants with accuracies of $26 \%$ and $43 \%$, respectively (see Table 2). Our algorithm-based classification using intrinsic connectivity from the resting brain (see Figure 2) resulted in superior accuracies for both male (52\%) and female (63\%) participants (see Table 2). Note that the chance probability of correct classification, assuming 4 groups, was $25 \%$. This classification performance provides a validity check for our automatic gender detection, showing that we can accurately classify biological sex based on our resting-state fMRI scans. The brain-derived gender signatures thus allowed for classification performances that exceeded the normative level of a widely used behavioral questionnaire for gender identity. 


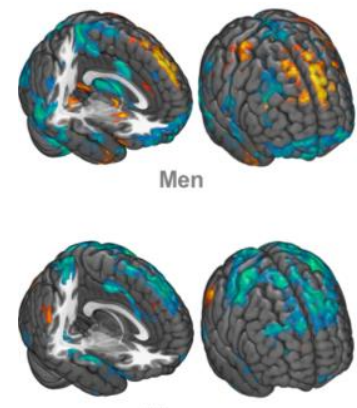

Women
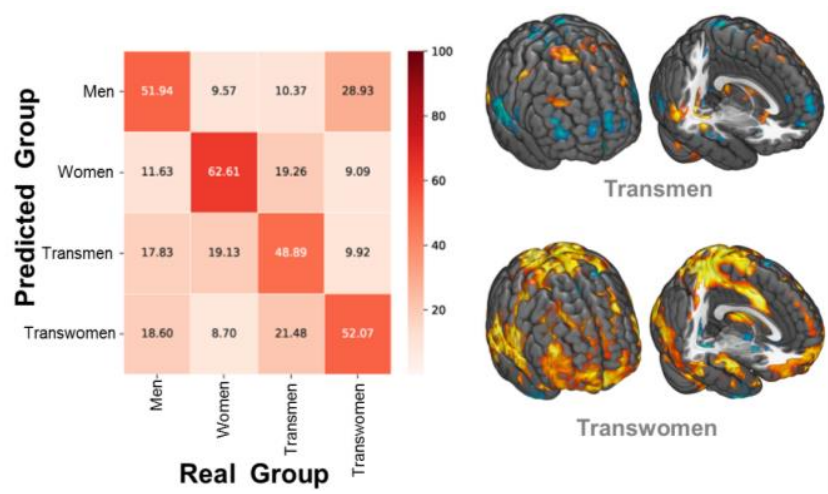

Transmen

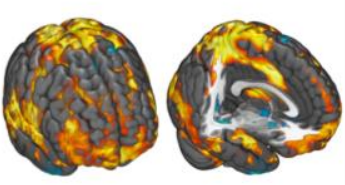

Transwomen

Real Group

Figure 2 - Men, women, trans men and trans women can be detected from brain connectivity

Each brain rendering shows how local grey-matter pattern contribute to predictive a given sub-type (e.g., man), relative to the three remaining sub-types. The confusion matrix in the middle indicates the variability of sub-group predictions, and that each is roughly equally mistaken in falsely predicting other sub-groups. SVM algorithms successfully distinguished all four gender groups based on intrinsic functional activity alone. Transgender participants were not preferentially classified as either men or women. As such, these two gender groups were uniquely defined in brain biology rather than representing mere variants of male or female brain activity signatures. All numbers inside the cells represent percentages of correctly classifying a particular group member as that group or misclassifying as one of the three other groups. The horizontal axis lists the psychological gender indicated by participants; the vertical axis lists the groups as predicted by the SVM algorithm. The discriminative classifier weights in the whole brain are rendered on the MNI-152 template for each group. Red-to-yellow colors indicate brain regions from which the SVM algorithm could best detect a given psychological gender. Blue-to-green colors indicate brain regions from which the SVM classifier build confidence against that particular self-reported gender. Lateral pre-motor and superior frontal areas had high predictive strength for male participants and vice versa for female participants. For trans women, discriminative regions covered large parts of temporal, parietal, occipital, frontal and sub-cortical midline areas. The brain signature for trans men on the other hand was confined to a small 
number of areas located in the posterior part of the temporal gyrus, the pre-motor cortex and the middle frontal gyrus.

Table 2 - Clinical and algorithm-based gender classifications of our sample

\begin{tabular}{|l|c|c|c|c|}
\hline & Female (F) & Male (M) & Trans men (Tm) & $\begin{array}{c}\text { Trans women } \\
\text { (Tw) }\end{array}$ \\
\hline $\begin{array}{l}\text { BSRI classification } \\
\text { accuracy per group }\end{array}$ & $43 \%$ & $26 \%$ & n.a. & n.a. \\
\hline $\begin{array}{l}\text { SVM classification } \\
\text { accuracy per group }\end{array}$ & $63 \%$ & $52 \%$ & $49 \%$ & $52 \%$ \\
\hline
\end{tabular}

The table summarizes the accuracy with which subjects were classified as either male or female for both the BSRI and the support vector machine (SVM) procedure. Whereas the BSRI classification was solely based on self-reports, all SVM classification results are based on resting-state functional connectivity data. As the BSRI does not allow for classification of trans men or trans women, these cells remain empty.

To seek neurobiological manifestations of gender identity subtypes beyond the common gender dichotomization, we were interested in how well the machine-learning algorithm was able to predict the two transgender groups. In accordance with our hypothesis of intermediate gender brain-types, we successfully detected both trans men and trans women as neurobiologically distinct phenotypes. Classification accuracies were $49 \%$ for trans men and $52 \%$ for trans women (see Figure $2 \&$ Table 2), compared to the random guessing probability of $25 \%$ each. In other words, transgender individuals were not systematically misclassified as skewed towards male or female. The brain-derived gender signatures (see Figure 2) show that each of the four groups is associated with a distinct, non-overlapping pattern of discriminative brain regions as determined by the support-vector classifier. These areas designate the parts of the brain where the algorithm could best classify the respective groups. 
A crucial aspect of these findings is that each of the four gender groups in our sample could be associated with distinct and non-overlapping brain connectivity patterns that differentiate them from the other three groups. Men and women exhibited largely distinct patterns for the superior frontal gyri and premotor areas: in our data set, these areas are highly predictive for correctly disambiguating male participants and the opposite is the case for female participants. Females are best predicted by an area located in the inferior parietal lobe. Successful prediction of trans men is associated with a few areas located close to the posterior part of the temporal gyrus, the pre-motor cortex and the middle frontal gyrus. Trans women on the other hand were best identified based on a widespread pattern involving temporal, parietal, occipital and frontal areas.

The next step in our analyses was to use canonical correlation analyses (CCA) to delineate multivariate relationships between functional connectivity and an array of gendercharacterizing behavioral traits from the BSRI across all 92 participants. CCA extends the idea of principal component analysis to two data matrices. We want to find 2 one-dimensional projections of the two variable sets such that the correlation between these two variables is maximized. The aim was to extract brain phenotypes for gender and transgender directly from the data. These phenotypes should allow us to delineate the brain-based gender continuum of individuals, irrespective of their physical sex characteristics. The CCA procedure was completely naive to the a-priori assignment of each participant to one of the four gender groups. Based on the statistically significant co-variance explained, the first nine canonical dimensions reached significance $(\mathrm{p}<0.001$; non-parametric permutation test, c.f. methods). Each one of these nine canonical dimensions represented a distinct pattern that associates a weighted set of BSRI responses with a weighted set of functional connections (see Figure 3). Thus, without using information on the particular group of a given participant, we were able to specify nine canonical dimensions, or brain-gender axes, that are sensitive to differences between cisgender 
and transgender individuals. How these nine brain-gender axes relate to both patterns of restingstate functional connectivity and BSRI response patterns is depicted in Figure 3.
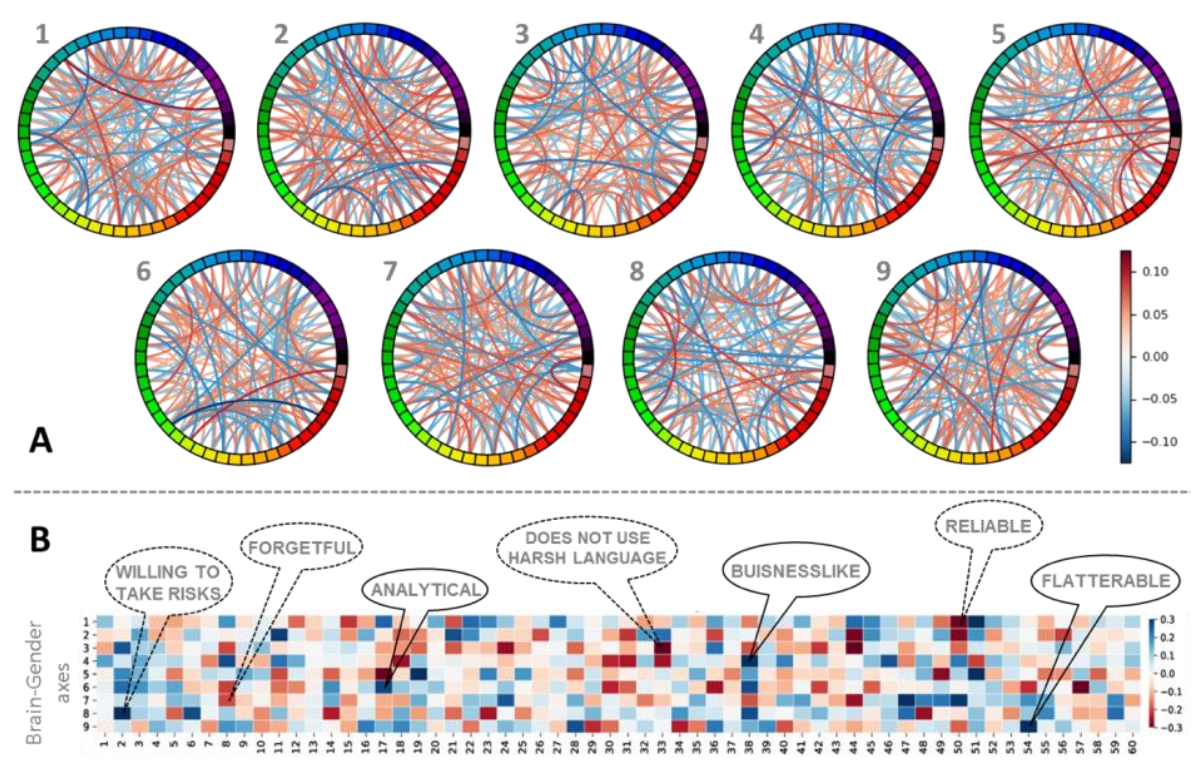

BSRI items

Figure 3 - Brain-gender axes revealed across cisgender and transgender participants

To test the feasibility of exploring continuous brain-behavior associations underlying gender variability more broadly, nine brain-gender axes (i.e., canonical vectors) are derived from the CCA. Here, we depict how these axes relate to both patterns of resting-state functional connectivity (panel A) and BSRI response patterns (panel B). The present brain-gender associations offer a gradual representation jointly describing cismen, ciswomen, trans men, and trans women. By combined analysis of BSRI data and resting-state functional connectivity, we define continuous dimensions underlying brain gender phenotypes. Notably, this CCA approach was naive to the gender group of each of the 92 participants, which was only used in the depicted post-hoc associations. (A): The nodes of the circle indicate the 50 spatial-temporally coherent brain networks extracted by ICA. Their edges indicate the positive or negative importance of a particular network-network coupling for each of the 9 brain-gender axes. The 
revealed nine brain-gender axes represent continuous degrees expressed in each of the 92 participants that transcended the four a-priori gender groups regarding their relation to the 50 different brain networks. Each of the nine brain-gender axes was associated with a specific pattern of connectivity among the 50 resting-state networks extracted via ICA. The colors on the circle indicate ICA nodes, whose mutual functional correlations were fed into the CCA procedure. Red (blue) color indicates increased (decreased) connectivity among two networks. (B): The relevance of 60 gender-related items is shown with their relation to the 9 brain-gender axes. The nine brain-gender axes represent continuous degrees expressed in each of the 92 participants that transcended the four a-priori gender groups regarding their relation to the 60 items of the BSRI questionnaire of gender-typical characteristics. Blue (red) color indicates a positive (negative) association of the specific item with a certain brain-gender axis. 


\section{Discussion}

Our results support the conclusion that the two existing categories of men and women are insufficient to be mapped to actual, modern-day gender identity categories. An individual's gender identity is one of the most important determinants of professional and personal life trajectories in human societies. Gender distinctions influence modern-day societies with respect to income levels, leadership, participation, health and academic status. Not conforming to the socially established gender norms might likely mean to face stigma, social exclusion and discriminatory practices, which in turn can have detrimental effects on physical and mental health. Specifically, many previous studies examining brain correlates of transgender tended to neglect the possibility of a dimensional gender construct, thus reinforcing a sexually dimorphic view of gender identity. This is worrisome, considering the steep increase in prevalence of gender incongruence and the associated social, psychological and monetary burdens for the affected individuals and our health care system.

The present study provides important quantitative evidence for future decision making in a clinical or societal context. We substantiated the existence of specific intermediate phenotypes for gender identity: besides male and female participants, our support-vector classifier was able to predict both trans women and trans men as distinct gender groups, with prediction accuracies above chance level for all four groups. Transgender sub-groups were identified with prediction accuracies comparable to men and women and were not systematically misclassified as skewed towards male or female. These results clearly support a dimensional gender perspective that captures four distinguishable brain phenotypes for gender identity. And since trans women and trans men were not primarily 'misclassified' as either men or women, these two groups constitute distinct gender subtypes which are associated with unique brain phenotypes. Thus, applying a simple, binary gender variable may incur an oversimplification that does not accurately capture the existing spectrum of human gender 
identities. Importantly, behavioral studies revealed that gender categorizations resulting from the BSRI correlate with various stereotypically gendered behaviors (Lee 1982; Hoffman and Borders 2001). Despite being widely employed for over 30 years now, the BSRI has also been criticized with respect to its underlying theoretical rationale, scoring methods and the item selection procedure (Hoffman and Borders 2001). Most critics pertained to the idea of labeling individuals solely based on BSRI scales: drawing conclusions merely on this measure might result in highly heterogeneous results and false conclusions. We suggest that this is irrelevant in the present study, due to the following reason: in our approach, the BSRI is not used to make definite predictions regarding male or female categorizations. We rather try to do the opposite: by gathering BSRI data from participants in addition to neurobiological measures and then combining the two variable sets, we explicitly do not rely on a single questionnaire. And despite some criticism, the BSRI is still considered to be a valid measure of gender identity and has undoubtedly provided useful input for research and discussion related to gender-related constructs and stereotypes (Beere 1990; Hoffman and Borders 2001). The BSRI has become the method of choice for the majority of researchers studying gender identity and has proven effective in different cultural settings (Hoffman and Borders 2001).

One thing that was not taken into account in our analyses was the influence of hormonal treatment and the menstrual cycle on the observed differences in resting-state networks. It has been shown in previous studies by our group and another lab (Van Goozen et al. 2002; Junger et al. 2014) that the presence or absence of hormonal treatment and the menstrual cycle do not substantially affect functional brain connectivity. Thus, even when explicitly controlling for hormonal/menstrual cycle status, no differences between gender dysphoric individuals were observed. On the other hand, there are studies which found hormonal effects on resting-state networks. These effects were only present for Tm and not Tw, and they were spatially confined to the frontal cortex and the cerebellum (Mueller et al. 2017). In a different study comparing 
Tm and cis-controls, significant changes due to hormonal treatment in functional connectivity between parietal and frontal regions have been reported (Burke et al., 2018). Differences in measures of resting-state functional connectivity due to hormonal effects thus seem to be more prominent in Tm and rather subtle, if present at all. Understanding the exact nature of such mechanisms will be an important goal for future studies, at best employing multimodal, longitudinal approaches in comprehensive samples containing both Tm and Tw. Furthermore, we acknowledge that future studies should gather additional information regarding recognition of gender incongruence, sexual orientation of subjects as well as beginning and exact nature of treatment. These factors might further influence brain phenotypes underlying gender identity.

In contrast to our fMRI connectivity investigation, many previous neuroimaging studies on gender identity focused on differences in brain volume. Overall, the results from studies investigating structural neuroanatomy are highly discrepant, mostly centered on a single or a small number of brain regions and in many cases even contradictory. Findings typically range from no differences between men and Tw (Savic and Arver, 2011), differences between Tw and both men and women (Luders et al., 2009), to an intermediate position for Tw between male and female brains (Rametti et al., 2011). Whereas the first two studies examined gray matter volumetric differences, Rametti and colleagues (2011) investigated fractional anisotropy (FA) of white matter fibers. Overall, these investigations indicate that specifically the hypothalamus and other subcortical structures seem to differ in either size or thickness between trans- and cisgender individuals. Investigating a different aspect of brain anatomy, other studies focused on comparing cortical thickness between transgender individuals and cisgender controls. Zubiaurre-Elorza and colleagues (2013) found evidence of subcortical gray matter masculinization for Tm and feminization in Tw, both right-lateralized. Luders and colleagues (2012) were also able to associate cortical thickness with gender identity, as they demonstrated thicker cortices in Tw, as compared to cis gender male controls, within several regions of the 
left and right hemisphere spread throughout the whole brain. Finally, Manzouri and Savic (2018) report multimodal imaging data, but focus mainly on cortical thickness, for a comprehensive sample of Tm, Tw, and hetero- as well as homosexual controls. For both Tm and Tw, they demonstrate increased cortical thickness as well as weaker structural and functional connections in the anterior cingulate-precuneus and right occipito-parietal cortex, regions known for mediating self-body perception. The authors implicate that the higher proportion of homosexual individuals in transgender groups may influence brain differences and controlling or correcting for sexual orientation may have a substantial effect on neuroimaging results (Manzouri and Savic 2018).

The most common denominator across all these studies investigating brain anatomy is that Tm and Tw represent an intermediate phenotype which seems to shift further towards the desired gender throughout the course of cross-sex hormone therapy (Mueller, De Cuypere, et al. 2017). In light of our current and previous (Clemens et al. 2017) resting-state connectivity findings, we concur with this statement, corroborating the existence of intermediate gender phenotypes for Tm and Tw which cannot be grouped together with men and women with respect to brain anatomy and functional connectivity. Previous findings from fMRI studies investigating transgender are typically interpreted in strict relation to males and females, neglecting the possibility that Tm and Tw may be characterized by distinct signatures in the brain. Like structural MR studies, these task-based and resting-state fMRI studies provide a complex and often contradictory pattern of results, with activation similarities and dissimilarities between transgender individuals and their cisgender counterparts (Mueller, De Cuypere, et al. 2017).

According to neurobiological theories (Garcia-Falgueras and Swaab 2009; Mueller, De Cuypere, et al. 2017), a combined influence of incongruence between hormonal effects on physical sex characteristics and brain sex and multiple genetic factors might explain the origins 
of transgender. Thus, a discrepancy between brain and genital differentiation, caused by genetic and hormonal factors, plays an important role in explaining the etiology of transgender. However, with respect to genetic factors, it is important to point out that so far, compelling evidence for a single gene tied directly to transgender is missing. Most likely, phenotypes relating to transgender and gender incongruence arise from the expression of polygenic genotypes or multiple genes (Zucker et al. 2016). Furthermore, Guillamon and colleagues (2016) stressed the difference in brain morphology between homo- and heterosexual Tw. Based on cortical thickness, diffusion tensor imaging and postmortem studies, they suggest that the observed differences between homosexual Tw and Tm and cis male and cis female controls are due to differently timed cortical thinning in various brain regions for each group. This differential cortical thinning process might occur primarily due to atypical effects of sex hormones and associated metabolites. While this aspect of differential cortical development might be important for the development of gender incongruence, other approaches stressed the incongruence between perception of own body and self (Manzouri and Savic 2018). They state that especially the emergence of a masculine or feminine identity is strongly influenced by the early development of a female or male body-self-perception. Accordingly, the underlying neurobiological mechanisms for gender incongruence and transgender could be linked to cortico-subcortical networks mediating self-body perception, which might be influenced by certain developmental and acquired changes (Manzouri and Savic 2018). Taking into account these various theoretical approaches and our own results, we suggest that the neurobiological mechanisms underlying transgender involve complex brain networks processing body perception and other self-reflective cognitive functions. A mixture of developmental, hormonal and genetic factors, particularly during youth and adolescence, might in turn influence these networks, resulting in changes of both functional and structural connectivity. From a clinical standpoint, it would make most sense to assess whether brain phenotypes of transgender persons observed in this and other studies exhibit long-lasting changes when receiving hormonal 
treatment, checking whether a shift to a risk profile for certain brain-based pathologies occurs. Optimally, initiatives to further clarify this matter would incorporate longitudinal studies with pooled transgender samples and multimodal imaging as well as multivariate pattern classification.

Employing a complex machine-learning approach, we demonstrate the ability to predict gender identity based on brain-behavior phenotypes. The fact that it takes less than 90 minutes to assess the data and the wide-spread availability of MR machines makes this procedure more appealing. Using such data-driven methods in the future to gather additional information about the gender identity of an individual might yield more objective data than the often rather subjective clinical evaluation. The overall goal of implementing such machine-learning techniques in the classification of gender identity is to provide additional, neurobiologically validated information, that is not influenced by societal pressure or social desirability, for both clinicians and patients. We are sure that assessing and providing such information will provide important additional information for many individuals and their relatives who are dealing with gender identity related issues. The goal here is to help those affected by gender incongruence to make decisions in the light of all available evidence, using not only the present study but future studies that should aim at replicating and extending our results to larger samples. Thus, we do not proclaim that clinical evaluation and other standard diagnostic procedures in the context of transgender should be abandoned. We merely provide additional data to assist and optimize decision making for those affected by gender incongruence. The goal is to establish an accurate biological characterization of transgender and gender identity, which in turn might aid early identification and medical guidance of transgender individuals. This approach would represent a significant step towards data-driven diagnostic markers, taking into account neurobiological and behavioral differences in an integrative modeling approach. Such an approach is in line with the development of the National Institute of Mental Health's Research 
Domain Criteria, which seek to construct a biologically-grounded framework for transdiagnostic characterization of biological markers (Cuthbert and Insel 2013).

Here, we extend previous knowledge on human gender identity by showing that trans men and trans women represent separate and gradual gender identity subtypes using two machine-learning algorithms and brain imaging data. While we found at least 4 separable gender identity subtypes, it should be noted that this result might have been partly due to inherent limitations of our analytic approach and that additional gender identity subtypes might exist. Thus, we acknowledge that our results can only indicate first steps towards unraveling the brain basis of gender identity. Given the challenging recruitment of our unique sample, we are happy about a sufficient sample size that showed out-of-sample prediction accuracy well above chance level. While future studies will need to corroborate our findings, we have made first steps towards a more nuanced conceptualization of gender dimorphism. These first steps should help to move away from the binary gender concept, hence taking away the pressure from transgender and intersexual individuals to fit into one of two categories. We should re-define the way we think and speak about transgender and other conditions that are not conforming to traditional gender concepts prevalent in modern societies. 


\section{Acknowledgements, Funding, Author Contributions}

The authors thank Sabine Bröhr, Cordula Kemper, Maria Peters, David Weyer and Teresa Karrer for their assistance and support. Specifically, we are indebted to all self-help groups, web forums and participants, whose keen support and endurance made this study possible.

This study was supported by the German Research Foundation (DFG: HA 3202/7-1 and IRTG 1328), the Start program 34/13 and the Brain Imaging Facility of the Interdisciplinary Centre for Clinical Research of the Faculty of Medicine at RWTH Aachen University, Germany. DB was funded by the German Research Foundation (DFG, BZ2/2-1, BZ2/3-1, and BZ2/4-1; International Research Training Group IRTG2150), Amazon AWS Research Grant (2016 and 2017), the German National Merit Foundation, as well as the START-Program of the Faculty of Medicine (126/16) and Exploratory Research Space (OPSF449), RWTH Aachen.

The authors report no biomedical financial interests or potential conflicts of interest.

U.H., B.D., E.S., J.N., J.J., T.A. and F.S. conceived the study design and acquired funding. J.J, E.S. and B.C. were responsible for data acquisition. D.B., G.M. and B.C. analyzed the data. B.C. wrote the first draft of the manuscript and prepared the figures. All authors read, approved and contributed to the final version of the manuscript. 


\section{References}

American Psychiatric Association. 2013. Diagnostic and Statistical Manual of Mental Disorders. American Psychiatric Association.

Beere CA. 1990. Gender roles: A handbook of tests and measures. Greenwood Publishing Group.

Bem SL. 1974. The measurement of psychological androgyny. J Consult Clin Psychol.

Bem SL. 1977. On the utility of alternative procedures for assessing psychological androgyny. J Consult Clin Psychol. 45(2):196-205.

Biswal BB, Mennes M, Zuo X-N, Gohel S, Kelly C, Smith SM, Beckmann CF, Adelstein JS, Buckner RL, Colcombe S, et al. 2010. Toward discovery science of human brain function. Proc Natl Acad Sci. 107(10):4734 LP - 4739.

Burke SM, Manzouri AH, Dhejne C, Bergström K, Arver S, Feusner JD, Savic-Berglund I. 2018. Testosterone Effects on the Brain in Transgender Men. Cereb Cortex. 28(5):1582-1596.

Bzdok D, Varoquaux G, Grisel O, Eickenberg M, Poupon C, Thirion B. 2016. Formal models of the network co-occurrence underlying mental operations. PLoS Comput Biol. 12(6):e1004994.

Chai XJ, Castañón AN, Öngür D, Whitfield-Gabrieli S. 2012. Anticorrelations in resting state networks without global signal regression. Neuroimage. 59(2):1420-1428.

Clemens B, Junger J, Pauly K, Neulen J, Neuschaefer-Rube C, Frölich D, Mingoia G, Derntl B, Habel U. (2017). Male- to- female gender dysphoria: Gender- specific differences in resting- state networks. Brain Behav. e00691.

Cuthbert BN, Insel TR. 2013. Toward the future of psychiatric diagnosis: the seven pillars of RDoC. BMC Med. 11(1):126.

Dessens AB, Slijper FM, Drop SL. 2005. Gender dysphoria and gender change in chromosomal females with congenital adrenal hyperplasia. Arch Sex Behav. 34:389-397.

Flores AR, Herman JL, Gates GJ, Brown TNT. 2016. How many adults identify as transgender in the United States? The Williams Institute.

Garcia-Falgueras A, Swaab DF. 2008. A sex difference in the hypothalamic uncinate nucleus: relationship to gender identity. Brain 131(12):3132-3146.

Garcia-Falgueras A, Swaab DF. 2009. Sexual differentiation of the human brain in relation to gender identity and sexual orientation. Funct Neurol. 24(1):17-28.

Gomez-Gil E, Gomez A, Canizares S, Guillamon A, Rametti G, Esteva I, Vazquez A, Salamero-Baro M. 2012. Clinical Utility of the Bem Sex Role Inventory (BSRI) in the Spanish Transsexual and Nontranssexual Population. J Pers Assess. 94(3):304-309.

Guillamon A, Junque C, Gómez-Gil E. 2016. A review of the status of brain structure research in Transsexualism. Arch Sex Behav. 45(7):1615-1648. 
Van Goozen SHM, Slabbekoorn D, Gooren LJG, Sanders G, Cohen-Kettenis PT. 2002. Organizing and activating effects of sex hormones in homosexual transsexuals. Behav Neurosci. 116(6):982-988.

Hanson SJ, Halchenko YO. 2008. Brain reading using full brain support vector machines for object recognition: there is no "face" identification area. Neural Comput. 20:486-503.

Hastie T, Tibshirani R, Friedman J. 2001. The Elements of Statistical Learning. Springer Series in Statistics, Heidelberg, Germany.

Herman-Jeglinska A, Grabowska A, Dulko S. 2002. Masculinity, femininity, and transsexualism. Arch Sex Behav. 31:527-534.

Heylens G, De Cuypere G, Zucker KJ, et al. 2012. Gender identity disorder in twins: a review of the case report literature. J Sex Med. 9:751-757.

Hoffman RM, Borders LD. 2001. Twenty-five years after the Bem Sex-Role Inventory: A reassessment and new issues regarding classification variability. Meas Eval Couns Dev. 34(1):39.

Jaffee KD, Shires DA, Stroumsa D. 2016. Discrimination and delayed health care among transgender women and men: implications for improving medical education and health care delivery. Med Care. 54:1010-1016.

Jenkinson M, Bannister P, Brady M, Smith S. 2002. Improved optimization for the robust and accurate linear registration and motion correction of brain images. Neuroimage. 17(2):825841.

Jenkinson M, Smith S. 2001. A global optimisation method for robust affine registration of brain images. Med Image Anal. 5(2):143-156.

Joel D, Berman Z, Tavor I, Wexler N, Gaber O, Stein Y, Shefi N, Pool J, Urchs S, Margulies DS, et al. 2015. Sex beyond the genitalia: The human brain mosaic. Proc Natl Acad Sci. 112(50):15468 LP - 15473.

Junger J, Habel U, Bröhr S, Neulen J, Neuschaefer-Rube C, Birkholz P, Kohler C, Schneider F, Derntl B, Pauly K. 2014. More than Just Two Sexes: The Neural Correlates of Voice Gender Perception in Gender Dysphoria. Fink B, editor. PLoS One. 9(11):e111672.

Kernbach JM, Yeo BTT, Smallwood J, Margulies DS, de Schotten MT, Walter H, Sabuncu MR, Holmes AJ, Gramfort A, Varoquaux G. 2018. Subspecialization within default mode nodes characterized in 10,000 UK Biobank participants. Proc Natl Acad Sci. 115(48):1229512300 .

Lee AG. 1982. Psychological Androgyny and Social Desirability. J Pers Assess. 46(2):147152.

Luders E, Sanchez FJ, Gaser C, Toga AW, Narr KL, Hamilton LS, Vilain E. 2009. Regional gray matter variation in male-to-female transsexualism. Neuroimage. 46(4):904-907. 
Luders E, Sanchez FJ, Tosun D, Shattuck DW, Gaser C, Vilain E, Toga AW. 2012. Increased Cortical Thickness in Male-to-Female Transsexualism. J Behav Brain Sci. 2(3):357-362.

Mazur T. (2005). Gender dysphoria and gender change in androgen insensitivity or micropenis. Arch Sex Behav. 34:411-421.

Mędraś M, Jóźków P. 2010. Transsexualism-diagnostic and therapeutic aspects. Endokrynol Pol. 61(4):412-416.

Manzouri A, Kosidou K, Savic I. 2017. Anatomical and Functional Findings in Female-toMale Transsexuals: Testing a New Hypothesis. Cereb. Cortex 27(2):998-1010.

Manzouri A, Savic I. 2018. Possible Neurobiological Underpinnings of Homosexuality and Gender Dysphoria. Cereb. Cortex doi: 10.1093/cercor/bhy090

Miller KL, Alfaro-Almagro F, Bangerter NK, Thomas DL, Yacoub E, Xu J, Bartsch AJ, Jbabdi S, Sotiropoulos SN, Andersson JLR, et al. 2016. Multimodal population brain imaging in the UK Biobank prospective epidemiological study. Nat Neurosci. 19(11):1523-1536.

Mueller SC, De Cuypere G, T'Sjoen G. 2017. Transgender Research in the 21st Century: A Selective Critical Review From a Neurocognitive Perspective. Am J Psychiatry.

174(12):1155-1162.

Mueller SC, Landré L, Wierckx K, T’Sjoen G. 2017. A Structural Magnetic Resonance Imaging Study in Transgender Persons on Cross-Sex Hormone Therapy. Neuroendocrinology. 105(2):123-130.

Oldfield RC. 1971. The assessment and analysis of handedness: the Edinburgh inventory. Neuropsychologia. 9(1):97-113.

Rametti G, Carrillo B, Gomez-Gil E, Junque C, Zubiarre-Elorza L, Segovia S, Gomez A, Guillamon A. 2011. The microstructure of white matter in male to female transsexuals before cross-sex hormonal treatment. A DTI study. J. Psychiatr. Res. 45(7):949-954.

Reed GM, Drescher J, Krueger RB, Atalla E, Cochran SD, First MB, Cohen-Kettenis PT, Arango-de Montis I, Parish SJ, Cottler S et al. 2016. Disorders related to sexuality and gender identity in the ICD-11: revising the ICD-10 classification based on current scientific evidence, best clinical practices, and human rights considerations. World Psychiatry 15(3):205-221.

Reisner SL, Poteat T, Keatley J, et al. 2016. Global health burden and needs of transgender populations: a review. Lancet 388:412-436.

Safer JD, Tangpricha V. 2008. Out of the shadows: it is time to mainstream treatment for transgender patients. Endocr Pract. 14:248-250.

Safer JD, Tangpricha V. 2019. Care of the Transgender Patient. Ann Intern Med. 171:ITC1ITC16.

Saraswat A, Weinand JD, Safer JD. 2015. Evidence supporting the biologic nature of gender identity. Endocr Pract. 21:199-204. 
Satterthwaite TD, Elliott MA, Gerraty RT, Ruparel K, Loughead J, Calkins ME, Eickhoff SB, Hakonarson H, Gur RC, Gur RE. 2013. An improved framework for confound regression and filtering for control of motion artifact in the preprocessing of resting-state functional connectivity data. Neuroimage. 64:240-256.

Savic I, Arver S. 2011. Sex Dimorphism of the Brain in Male-to-Female Transsexuals. Cereb Cortex. 21(11):2525-2533.

Schneider-Düker M, Kohler A. 1988. Assessment of sex roles: Results of a German version of the Bem Sex-Role Inventory. Diagnostica. 34(3):256-270.

Smith ES, Junger J, Derntl B, Habel U. 2015. The transsexual brain-A review of findings on the neural basis of transsexualism. Neurosci Biobehav Rev. 59:251-266.

Smith SM. 2002. Fast robust automated brain extraction. Hum Brain Mapp. 17(3):143-155.

Smith SM, Fox PT, Miller KL, Glahn DC, Fox PM, Mackay CE, Filippini N, Watkins KE, Toro R, Laird AR, et al. 2009. Correspondence of the brain's functional architecture during activation and rest. Proc Natl Acad Sci. 106(31):13040 LP - 13045.

Smith SM, Nichols TE, Vidaurre D, Winkler AM, Behrens TEJ, Glasser MF, Ugurbil K, Barch DM, Van Essen DC, Miller KL. 2015. A positive-negative mode of population covariation links brain connectivity, demographics and behavior. Nat Neurosci. 18(11):15651567.

Wittchen HU, Zaudig M, Fydrich T. Strukturiertes klinisches interview für DSM-IV. 1997. Göttingen: Hogrefe.

Witten DM, Tibshirani R, Hastie T. 2009. A penalized matrix decomposition, with applications to sparse principal components and canonical correlation analysis. Biostatistics. 10(3):515-534.

Zhou JN, Hofman MA, Gooren LJ, Swaab DF. (1995). A sex difference in the human brain and its relation to transsexuality. Nature 378:68-70.

Zubiaurre-Elorza L, Junque C, Gómez-Gil E, Segovia S, Carrillo B, Rametti G, Guillamon A. 2013. Cortical Thickness in Untreated Transsexuals. Cereb Cortex. 23(12):2855-2862.

Zucker KJ, Lawrence AA, Kreukels BPC. 2016. Gender Dysphoria in Adults. Annu Rev Clin Psychol. 12(1):217-247. 